\title{
Resting State fMRI as A Tool for the Study of Consciousness
}

\author{
Tomás Labbé Atenas* and Javiera Venegas Bustos \\ School of Medicine, University of Santiago, Chile
}

Received: 制 January 22, 2019; Published: 䟧 January 31, 2019

*Corresponding author: Tomas Labbé Atenas, MD, PhD, School of Medicine, University of Santiago, Chile

\begin{abstract}
Consciousness constitutes a complex concept. Its clinical and phenomenological definitions are commonly under debate. Resting- State functional Magnetic Resonance Imaging has contributed to the understanding of different cognitive processes by providing neural mechanisms underlying specific functions. Resting State acquisitions has shown an important role of Resting State networks both in different mental state and consciousness alterations as those disorders of consciousness observed in neurological pathologies and specific states as drug-induced hallucinations and traumatic brain injury. This evidence situates the complex focus of this article, the consciousness, as an accurate balance between activity and connectivity of regions and neural networks.
\end{abstract}

Keywords: Neuroimaging; Resting State fMRI; Consciousness; Disorders of Consciousness; Hallucinations; Default Mode Network

Abbreviations: DOC: Disorder of Consciousness; RSN: Resting State Networks; DMN: Default Mode Network

\section{Introduction}

The mere effort to provide an acute definition of consciousness constitutes a major challenge in neurosciences and the conventional characterizations of this concept are currently under strong debate. Nevertheless, several approaches have been developed in order to answer the main question of how the subjective experience emerges. Diverging models, from complex psychological explanations to the denial of any relevance of the consciousness, limiting it strictly to an epiphenomenon of the disposition to act have been discussed in the scientific community. The clinical characterization of this complex concept often considers two main axes. In the first place, arousal is a quantitative approach by which a subject is described as asleep or awake. On the other hand, awareness is a qualitative approach related to the content of the subjective experience. This differentiation has influenced the research-oriented to look for neural correlates of consciousness [1,2].

Figure 1 shows the interacción between these complementary axes, exhibiting examples of conditions in each quadrant. Nevertheless, the balance between arousal and awareness is a poor approach to human conscious experience. There are a lot of clinical and nonclinical scenarios in which consciousness is under a temporary or permanent modification. For example, specific mental states as the exhibited in meditation have been studied and brain activity and connectivity changes related to the onset of meditative activity have been identified [3-5].

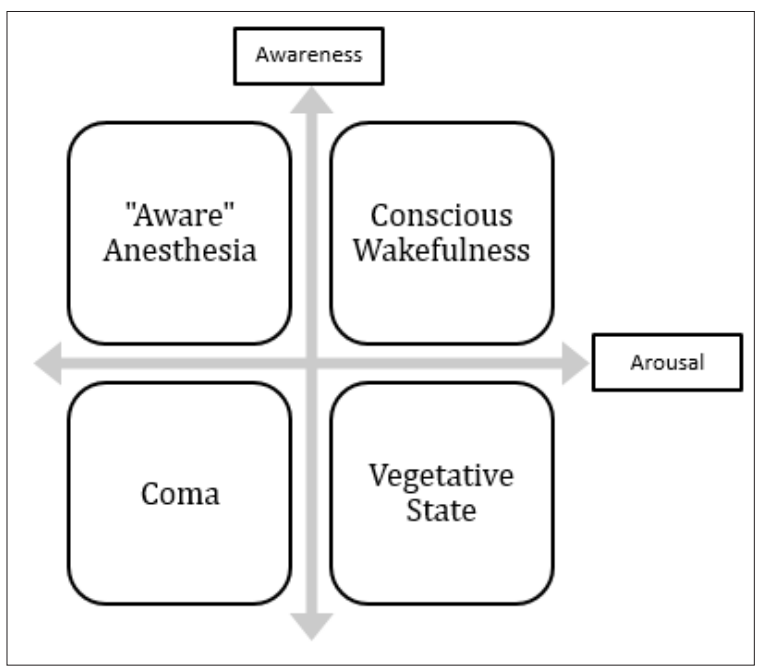

Figure 1: Consciousness as the complex clinical expression of arousal and awareness. Vertical axis representing awareness show lower level at the bottom and higher level at the top. Horizontal axis representing shows lower level at the left and higher level of arousal at the right side. Examples exhibited in each quadrant are no exhaustive, because much more options may be found in the clinical progression of arousal and awareness in both directions. 
Despite the existence of those differential definitions and methodological approaches, a common component among the predominant models is the association between the states of consciousness to specific neural correlates [6]. The study of large scale neuronal processes in the human brain during resting state fMRI has contributed to understanding the mechanisms underlying to several cognitive domains and related to both normal and altered conscious experience [7]. Common and daily states of the human attentional state as mind-wandering has also been related to functional connectivity changes observed during resting state fMRI acquisitions [8]. Figure 2 Shows a schematization of some identifiable resting state networks which have been related to specific cognitive domains. In this article, we review specific contexts of conscious models, as disorders of consciousness, anesthesia, hallucinations, and brain injury and we analyze how the restingstate fMRI has contributed to understanding their underlying mechanisms, also providing an insight into the neurodynamic characterization of the complex concept of consciousness.

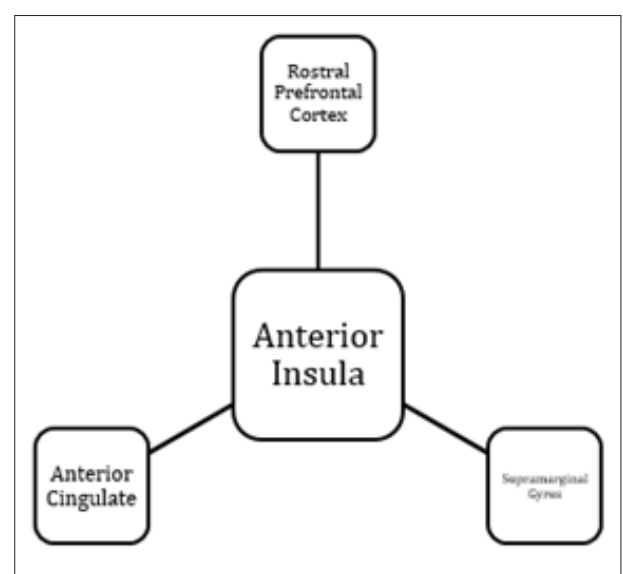

Figure 2: Schematization of a Resting State Network. During the absence of explicit cognitive activity, brain areas maintain preferential connectivity measurable by resting-state fMRI. In these conditions, an identifiable region can act as a core of the information flow between areas being denominated as a Hub. In this schema (similar to Saliency Network) anterior Insula is focusing most of the connectivity and influencing the neural activity of the other shown regions.

\section{Disorders of Consciousness}

Disorder of Consciousness (DOC) is a critical component of several neurological conditions. Despite its categorization as vegetative state, minimally conscious state or coma, connectivity has been found to be disrupted in different resting-state networks (RSN) [9]. Some RSN as the Default Mode Network (DMN) appears to play a central role in some DOC. While Unresponsive Wakefulness Syndrome has been strongly related with the functional disorganization of the Default Mode Network [10], some evidence suggests that the disorganization of the functional connectivity between mediodorsal thalamus and DMN may play an important role in the DOC [11]. This thalamocortical interaction has been identified as a substrate of the consciousness compromise in at least two specific ways, an externally oriented sensory-motor network, or extrinsic system, and an inward-oriented network, or intrinsic or default system. Specific compromises of these networks could explain different alterations of consciousness as absorption, a detachment of the external world or other clinically identifiable forms of DOC. Then, rsfMRI has emerged as an interesting tool in the prediction of cognitive state and recovery chances in pathologies including this kind of disorder [12]. However, thalamocortical changes are not the only responsible for changes in this external and internal balance. The abnormal activation of in insula, lingual gyrus and motor areas have shown to interrupt this balance [13].

\section{Anesthesia}

Interestingly, resting-state fMRI acquisitions have exhibited induced anesthesia, not as a unique interruption of brain activity. Conversely, specific neurodynamic findings have been related to different anesthetic drugs. In the case of Propofol, the induced anesthesia modifies both resting state neural activity and also brain networks related to specific cognitive functions [14]. Also, coactivation patterns of posterior Cingulate Cortex allowed identifying different states of consciousness during sedative induction [15-17]. On the other hand, sevofluraneinduced connectivity changes have been studied under fMRI and complementary methods as electroencephalogram and Positron Emission Tomography [18]. This anesthesia method modifies the connectivity between and within some RSN. Nevertheless, the activity of some networks remains unaltered under anesthesia effect [19]. Further analysis of the acquisitions in this condition shows that sevoflurane-induced unconsciousness is associated with both globally reduced BOLD signal and selectively reduced functional connectivity between DMN components, also reorienting the global connectivity to ventral attention network which is related to saliency [20].

Globally, the evidence derived from experimental designs related to anesthetic drugs has shown that neurodynamic of specific networks could explain conditions as Disconnected Consciousness, in which a complete sensory-motor disconnection from the environment occurs with preserved internal mentation [17]. Different measurements and technical approaches to BOLD signal have been evaluated in the study of human brain under sedation [21] and a deep insight into consciousness mechanisms can still be elucidated by this experimental approach.

\section{Hallucinations}

Hallucinations are a specific alteration of conscious content associated with the consumption of psychoactive drugs. An important amount of information derived of LSD-induced mental states links some changes in hub connectivity to the hallucinations (Muller, 2018). Also, alterations in thalamic and global brain connectivity appear to be correlated with altered conscious states in the consumption of this drug $[22,23]$. On the other hand, we may assume that any psychoactive drug will have identifiable effects over neurodynamic. For example, specific brain connectivity changes are described in relation to psychedelic ayahuasca [24]. 


\section{Brain Injury}

Resting state fMRI describes the widely distributed changes in brain connectivity observed in patients with diffuse axonal injury [25]. RSN connectivity changes persist during the recovering of traumatic coma, both in the area of the lesion and also in widely distributed regions [26]. In the longitudinal analysis, progressive an identifiable change is found by this technical approach in relation to consciousness level when acquired brain injury is evaluated [27]. A brain affected by diffuse brain injury exhibits both changes in functional connectivity and amplitude of low-frequency fluctuations. Interestingly some characteristics of resting-state acquisitions have shown to be related to better prognosis [28]. Then, fMRI has been proposed as a tool in acute brain injury in the very early period of intensive care unit admission [29], because of the relation between DMN connectivity findings and potential spontaneous recovery [30] and with the responsiveness to nonconventional management as transcranial stimulation [31].

\section{Conclusion}

Consciousness is not a monolithic concept. Much of its compromises can be assessed by considering two main axes, the arousal, and the awareness. Nevertheless, the objective of this article was not to provide an accurate characterization and an exhaustive description of the current debate around the conscious experience and it scientific and philosophical implications. Resting-state fMRI constitutes a useful tool to evaluate consciousness both at the clinical level and in the field of fundamental neuroscience. At different mental and conscious state, it provides an interesting description of how critical brain regions and specific networks are functionally connected and interacting to allow information flow between areas. The wide range of disorders of consciousness observed in different neurological conditions exhibits the preferential compromise of relevant RSN as DMN which is critically connected and influence over the global brain activity. Concrete contexts as meditation, transitory mental states, sedation or drug-induced hallucinations provide additional information on how normal consciousness appears to emerge of the balance between identifiable networks strongly related to important cognitive domains.

\section{References}

1. T Marvan, M Polak (2017) Unitary and dual models of phenomenal consciousness. Conscious Cogn 56: 1-12.

2. A Soddu, A Vanhaudenhuyse, A Demertzi, MA Bruno, L Tshibanda (2011) Resting state activity in patients with disorders of consciousness. Funct Neurol 26(1): 37-43.

3. KB Baerentsen (2015) Patanjali and neuroscientific research on meditation. Frontiers in psychology 6: 915.

4. KB Baerentsen, H Stodkilde Jorgensen, B Sommerlund, T Hartmann, J Damsgaard Madsen (2010) An investigation of brain processes supporting meditation. Cognitive processing 11(1): 57-84.

5. T Jao, CW Li, PE Vertes, CW Wu, S Achard (2016) Large-Scale Functional Brain Network Reorganization During Taoist Meditation. Brain connectivity 6(1): 9-24.

6. TS Meese (2018) The How and Why of Consciousness? Frontiers in psychology 9: 2173.
7. A Lundervold (2010) On consciousness, resting state fMRI, and neurodynamics. Nonlinear biomedical physics 4 (Suppl 1): S9.

8. A Kucyi, KD Davis (2014) Dynamic functional connectivity of the default mode network tracks daydreaming. NeuroImage 100: 471-480.

9. A Demertzi, F Gomez, JS Crone, A Vanhaudenhuyse, L Tshibanda (2014) Multiple fMRI system-level baseline connectivity is disrupted in patients with consciousness alterations. Cortex; a journal devoted to the study of the nervous system and behavior 52: 35-46.

10. C Di Perri, E Amico, L Heine, J Annen, C Martial (2018) Multifaceted brain networks reconfiguration in disorders of consciousness uncovered by co-activation patterns. Human brain mapping 39(1): 89-103.

11. JH He, Y Cui, M Song, Y Yang, YY Dang (2015) Decreased functional connectivity between the mediodorsal thalamus and default mode network in patients with disorders of consciousness. Acta neurologica Scandinavica 131(3): 145-151.

12. A Soddu, M Boly, Y Nir, Q Noirhomme, A Vanhaudenhuyse (2009) Reaching across the abyss: recent advances in functional magnetic resonance imaging and their potential relevance to disorders of consciousness. Progress in brain research 177: 261-274.

13. JH He, Y Yang, Y Zhang, SY Qiu, ZY Zhou (2014) Hyperactive external awareness against hypoactive internal awareness in disorders of consciousness using resting-state functional MRI: highlighting the involvement of visuo-motor modulation. NMR in biomedicine 27(8): 880-886.

14. Z Huang, J Zhang, J Wu, X Liu, J Xu (2018) Disrupted neural variability during propofol-induced sedation and unconsciousness. Human brain mappin 39(11): 4533-4544.

15. E Amico, F Gomez, C Di Perri, A Vanhaudenhuyse, D Lesenfants (2014) Posterior cingulate cortex-related co-activation patterns: A resting state FMRI study in propofol-induced loss of consciousness. PloS one 9(6): e100012.

16.X Liu, KK Lauer, BD Ward, CJ Roberts, S Liu (2017) Fine-Grained Parcellation of Brain Connectivity Improves Differentiation of States of Consciousness During Graded Propofol Sedation. Brain connectivity $7(6): 373-381$.

17. X Liu, KK Lauer, BD Ward, CJ Roberts, S Liu (2018) Regional entropy of functional imaging signals varies differently in sensory and cognitive systems during propofol-modulated loss and return of behavioral responsiveness. Brain imaging and behavior.

18. BJA Palanca, MS Avidan, GA Mashour (2017) Human neural correlates of sevoflurane-induced unconsciousness. British journal of anaesthesia 119(4): 573-582.

19. M Kafashan, S Ching, BJ Palanca (2016) Sevoflurane Alters Spatiotemporal Functional Connectivity Motifs That Link Resting-State Networks during Wakefulness. Frontiers in neural circuits 10: 107.

20. BJ Palanca, A Mitra, L Larson Prior, AZ Snyder, MS Avidan, et al. (2015) Resting-state Functional Magnetic Resonance Imaging Correlates of Sevoflurane-induced Unconsciousness. Anesthesiology 123(2): 346356.

21. AT Baria, MV Centeno, ME Ghantous, PC Chang, D Procissi, et al. (2018) BOLD temporal variability differentiates wakefulness from anesthesiainduced unconsciousness. Journal of neurophysiology 119(3): 834-848.

22. KH Preller, JB Burt JL Ji, CH Schleifer, BD Adkinson, P Stampfli, et al. (2018) Changes in global and thalamic brain connectivity in LSDinduced altered states of consciousness are attributable to the 5-HT2A receptor. ELife, pp: 7.

23. F Muller, C Lenz, P Dolder, U Lang, A Schmidt, et al. (2017) Increased thalamic resting-state connectivity as a core driver of LSD-induced hallucinations. Acta psychiatrica Scandinavica 136(6): 648-657. 
24. A Viol, F Palato Fontes, H Onias, DB de Araujo, GM Viswanathan (2017) Shannon entropy of brain functional complex networks under the influence of the psychedelic Ayahuasca. Scientific reports 7(1): 7388.

25. S Yao, J Song, L Gao, Y Yan, C Huang, et al. (2015) Thalamocortical Sensorimotor Circuit Damage Associated with Disorders of Consciousness for Diffuse Axonal Injury Patients. Journal of the neurological sciences 356(1-2): 168-174.

26. S Lee, JR Polimeni, CM Price, BL Edlow, JA McNab (2018) Characterizing Signals Within Lesions and Mapping Brain Network Connectivity After Traumatic Axonal Injury: A 7 Tesla Resting-State FMRI Study. Brain connectivity 8(5): 288-298.

27. Q Zou, X Wu, J Hu, W Tang, Y Mao, et al. (2017) Longitudinal recovery of local neuronal activity and consciousness level in acquired brain injury. Human brain mapping.

\section{ISSN: 2574-1241}

DOI: $10.26717 / B J S T R .2019 .13 .002469$

Tomás Labbé Atenas. Biomed J Sci \& Tech Res

(c) (i) This work is licensed under Creative

Submission Link: https://biomedres.us/submit-manuscript.php
28. H Guo, R Liu, Z Sun, B Liu, Y Xiang, et al. (2018) Evaluation of Prognosis in Patients with Severe Traumatic Brain Injury Using Resting-State Functional Magnetic Resonance Imaging. World neurosurgery 121: e630-e639.

29. D Kondziella, PM Fisher, VA Larsen, J Hauerberg, M Fabricius, et al. (2017) Functional MRI for Assessment of the Default Mode Network in Acute Brain Injury. Neurocritical care 27(3): 401-406.

30. ZD Threlkeld, YG Bodien, ES Rosenthal, JT Giacino, A Nieto Castanon, et al. (2018) Functional networks reemerge during recovery of consciousness after acute severe traumatic brain injury. Cortex; a journal devoted to the study of the nervous system and behavior 106: 299-308.

31. C Cavaliere, M Aiello, C Di Perri, E Amico, C Martial, et al. (2016) Functional Connectivity Substrates for TDCS Response in Minimally Conscious State Patients. Frontiers in cellular neuroscience 10: 257.

$\begin{array}{ll}\text { BIOMEDICAL } & \text { Assets of Publishing with us } \\ \text { RESEARCHES } & \text { - Global archiving of articles } \\ \text { - Immediate, unrestricted online access } \\ \text { https://biomedres.us/ }\end{array}$

